\title{
Prevalence and characteristics of sexual harassment among high school students: a pilot study
}

\author{
Greta Grigentyte and Sigita Lesinskiene* \\ Clinic of Psychiatry, Institute of Clinical Medicine, Faculty of Medicine, Vilnius University, Lithuania
}

\begin{abstract}
Background: Although sexual harassment among adolescents is a current major concern, there is little data on its prevalence and characteristics that might be helpful to improve preventive programs at schools.

Aim: The purpose of our study to evaluate the prevalence, predominant forms and perpetrators of sexual harassment, common places of the incidents and subjective estimations of influence on mood, sleep and eating habits among high school students aged 16 to 19.

Methods: The questionnaire has been created for research purpose by the authors, it consists of five main sections, which are: a demographic section, a section about unwanted and unpleasant verbal sexual harassment, a section about unwanted sexual gestures, a section of unwanted and unpleasant physical sexual harassment, and a section of subjective evaluations of impact on students' mood, sleep and eating habits.

Results: 209 students participated in the research: 65\% girls and 35\% boys. 56\% of participants indicated that they had experienced one of the three forms of sexual harassment (verbal, gestures or physical). We found significant difference between the genders: girls are facing unwelcome sexual behavior more frequently than boys do $(\mathrm{p}<0,001)$. The most common form of sexual harassment among adolescents was found to be sexual gestures. According to the findings, sexual gestures were repeated and frequent, while physical sexual behavior tended to be experienced once. The majority of students reported the harasser to be unknown person and the incidents often happened in the public places. $50 \%$ of participants reported that experienced sexual harassment had some impact on their mood, $11 \%$ reported having sleep disturbances and $9 \%$ - a change of eating habits.
\end{abstract}

\section{Introduction}

Sexual harassment is a topic of interest for more than two or three decades, but today its relevancy has developed into a powerful movement called \#metoo. This movement is a signal for researchers to collect more data on the phenomenon called sexual harassment, pointing out subjective experiences of the victims and especially vulnerable populations, which might be useful in later prevention programs.

Previous researches demonstrated that adolescents are in a high risk of becoming victims of sexual harassment, more often perpetrated by peers than by teachers or other adults [1-4]. Girls experience sexual harassment more often than boys do, furthermore, girls tend to experience sexual harassment qualitatively more severe and subjectively more negative [1,2,5-7].

According to AAUWEF report on sexual harassment at school, the majority of incidents are made up of verbal sexual harassments (unwelcome sexual comments, jokes, or gestures), but physical sexual harassment seems to be also an issue at schools [8]. The other important point is that more than a half of students experience sexual harassment more than once per school year. Around 9 percent of students indicate that a harasser was a teacher or other adult at school, however, many incidents remain untold and unknown [8].

It has been reported that sexual harassment at school might have short or even longer consequences for students' emotions and education, especially for girls', including decreased productivity and absenteeism from school [9]. Moreover, girls report more psychosomatic symptoms than boys do and also they show lower self-esteem levels compared to boys [4].

\begin{abstract}
Aim
The purpose of our study was to evaluate the prevalence, predominant forms and perpetrators of sexual harassment and the common places of the incidents among high school students aged 16 to 19 . Moreover, this research was carried out to find out what are the consequences of sexual harassment for high school students, which has been considered to be best evaluated by subjective quantitative and qualitative estimations of influence on victims' mood, sleep and eating habits.
\end{abstract}

\section{Methods}

\section{Participants}

209 second-fourth-year students at high school in Vilnius participated in the research. For this pilot study one school had been chosen due to its demographical convenience. We contacted the school office and received signed permission for our research. Parent informed

${ }^{\star}$ Correspondence to: Sigita Lesinskiene, Clinic of Psychiatry, Institute of Clinical Medicine, Faculty of Medicine, Vilnius University, Lithuania, Tel: +370686 17550; E-Mail: sigita.lesinskiene@mf.vu.lt

Key words: adolescents, high school students, sexual harassment, public place, sleep, mood, sexual gestures, physical harassment, verbal sexual harassment

Received: July 16, 2018; Accepted: July 26, 2018; Published: July 28, 2018 
consent forms were also used, which had been given for students to bring them home and have them signed before we performed the survey. Most parents signed informed consent forms signing "yes, I agree" and the majority of students were eager to participate in the survey, probably because the questionnaires were to be completed during lessons and the topic was popular and intriguing at that time. The problem we faced was that some of the students forgot to bring these forms so they were automatically excluded from the study.

The students were 16 to 19 years old. $65 \%$ participants were girls and 35\% were boys. There were only three spoiled questionnaires, which were not taken seriously, it might have been influenced by the fact that a researcher was in the classroom and answered the pupils' questions if there were any.

\section{Questionnaire}

The questionnaire has been created for research purpose by the authors and it consists of five main sections: a demographic section, a section about unwanted and unpleasant verbal sexual harassment, a section about unwanted sexual gestures, a section of unwanted and unpleasant physical sexual harassment and a section of subjective evaluations of impact on students' mood, sleep and eating habits. These three sections (verbal, gestures and physical harassment) were very similar, which made it much easier to complete the questionnaire, as we know that students nowadays tend to be more distracted and impatient. There were two types of questions: most of them were closed-end questions and one open-ended (qualitative) in each section. Respondents were asked to describe their unwanted and unpleasant sexual harassment experiences in more detail.

In all of the three main sections called "verbal sexual harassment", "sexual gestures" and "physical sexual harassment" participants were asked if ever experienced that form of harassment (yes/no/I do not know), if yes, they were asked to describe this experience in more detail, then asked if it happened once, often or continually, who was the perpetrator (a friend, other student, a teacher or other adult working at school, a parent or a family member, an unknown person or "other"), where the incident took place (at school, at home, in a public place, like on a bus, concert, etc. or "other") and to evaluate subjective feelings about the sexual harassment from 1 to 10 , when $1=$ a minimum unpleasant, $10=$ a maximum unpleasant incident.

The last section was about personal (or subjective) evaluation of impact on three main parts, which could be a reliable tool to quickly identify students who might need specific help after being harassed. These three parts were: mood, sleeping and eating. Open-ended questions were given to collect more detailed data on impact on the change of the mood, sleeping and eating habits.

Because of the known limitations of the term "sexual harassment" from the previous researches among adolescents [4], adjectives "unwanted" and "unpleasant" were used before the name sexual harassment, which were bolded, and also specific examples were given in the brackets, e.g." "Have you ever experienced unwelcome, unpleasant comments, jokes or bullying of sexual nature, what you personally evaluate as to be sexual harassment? (E.g., unpleasant sexual joke or story, unwanted calling "baby", "honey", unpleasant sexual comments of your appearance and look, asking of your sexual fantasies, etc.)".

\section{Results}

209 students participated in the research, $136(65 \%)$ girls and 73 (35\%) boys. The mean age was $17,4(\mathrm{SD}=0,936) .56 \%$ of participants indicated that they had experienced one of the three forms of sexual harassment (verbal, gestures or physical) (Table 1). Among participating girls $76 \%$ reported experience of sexual harassment and among boys - $18 \%$. We found statistically significantly difference between different genders: girls are facing unwelcome and unwanted sexual behavior [3] more frequently than boys do $(\mathrm{p}<0,001)$.

Depending on form, the most frequent form of sexual harassment among adolescents was found to be sexual gestures (Table 2). $74 \%$ of those, who indicated being harassed, noted experiencing sexual gestures, $62 \%$ - verbal comments and 57\% - unwanted and unwelcome physical sexual behavior. On the other hand, depending on the gender, results were different: among girls the most frequent form of sexual harassment was sexual gestures, but among the boys - sexual comments, jokes and bullying (Table 3).

Typical examples of unwelcome and unpleasant sexual harassment reported by the respondents were collected. Sexually suggestive comments were found to be the most popular verbal sexual harassment form, here are few examples from the collected data:

When I was going home by train, a man, who was also a passenger, told me that my butt was attractive to him and that he would make love to me if I was older.

When I was going home I heard few comments from the neighbor house terrace calling me to come and play.

When walking on the street wearing shorts, skirt or open neck $t$-shirt I hear phrases commenting my body, especially breasts, buttock, and offers to give them kisses or to go out with them.

Physical forms of sexual harassment were described by respondents typically giving more details about the perpetrators and places of the incidents:

When I was on a ferry to Sweden, a foreigner man put his hand on my buttocks and soon went away.

Walking a corridor at school a boy grasped my breasts.

During previous years at school boys used to grasp buttocks, etc., I hardly could run further from them.

I was going by bus and an unknown man started to touch and stroke my thigh.

As mentioned above, the most common form of sexual harassment was found to be sexual gestures. Here are few examples described by the respondents:

Table 1. Incidence of sexual harassment by gender, in percentage $(\%)(\mathrm{N}=209), \mathrm{p}<0,001$

\begin{tabular}{|l|c|}
\hline Girls & 76 \\
\hline Boys & 18 \\
\hline Total & 56 \\
\hline
\end{tabular}

Table 2. Types of sexual harassment, in percentage (\%) $(\mathrm{N}=116)$

\begin{tabular}{|c|c|}
\hline Verbal & 62 \\
\hline Gestures & 74 \\
\hline Physical & 57 \\
\hline
\end{tabular}

Table 3. Types of sexual harassment by gender, in percentage (\%) $(\mathrm{N}=116)$

\begin{tabular}{|c|c|c|}
\hline Verbal & 62 & 62 \\
\hline Gestures & 79 & 38 \\
\hline Physical & 60 & 31 \\
\hline
\end{tabular}


At party or even at school girls whom I hardly know, address me by unwelcome, vulgar gestures.

Staring, looking me up and down, catcalls, unpleasant coming closer, following on the street is a common behavior of strangers.

I was standing on a bus stop with my friend when a dizzy man came to us, started talking to us unkindly, me and my friend wanted to go away, but he started to follow us continually insulting.

In the questionnaire there was also a question if the unwelcome and unpleasant sexual behavior happened once, often or was continual. According to our findings, sexual gestures are repeated and frequent, while physical sexual behavior tend to be experienced once. Verbal sexual harassment experience depends on gender: girls indicated that it was a repeated behavior, whereas boys reported onetime experiences (Table 4).

Who were the perpetrators, mentioned by students? Having in mind previous researches, we predicted, that unwanted sexual behavior perpetrated by peers might be the most common type, however, empirical observations let us hypothesize that unknown people also perform sexual behaviors very often. As predicted, the percentage of respondents who reported unwelcome verbal, physical sexual behavior or sexual gestures by their peers was high - respectively $42 \%, 33 \%$ and $27 \%$. However, the majority of students mentioned the harasser to be an unknown person: $51 \%, 64 \%$ and $70 \%$ (Figure $1 \mathrm{~A}, 1 \mathrm{~B}, 1 \mathrm{C}$ ). This pilot study raises an issue that sexual harassment at home is another problem - $4 \%$ of respondents indicated that they have experienced verbal sexual comments, jokes or bullying, $2 \%$ - sexual gestures, $3 \%$ - physical sexual harassment by their family member.

When creating design of the research and preparing questionnaire, it was predicted that sexual harassment is a widely spread public behavior. We also wanted to explore if this behavior is frequent at home and at school. Not surprisingly, the majority of described incidents reported by participants took place in public places: on a bus, bus stop, at concerts, at cafes, in parks, etc. It involved more than a half of all the incidents (Figure 2). On the other hand, what was found and caused so much of concern was the prevalence of sexual harassment at home and at school: $4 \%$ unwelcome, unpleasant sexual comments, jokes took place at home and $16 \%$ - at school; $14 \%$ sexual gestures and $13 \%$ unwelcome physical sexual behavior have been reported happening at school; and at last - $8 \%$ participants have experienced physical sexual harassment at home.

As described in section "Methods", the participants were asked to evaluate their subjective feelings about the sexual harassment (each form, if experienced) from 1 to 10 . Evaluation of subjective impact on feelings differed between genders. Boys reported their subjective feelings about the verbal harassment from minimum 2 to maximum 8 points out of 10 and the median was 2,0 , whereas girls' subjective

Table 4. Frequency of sexual harassment by gender, in percentage $(\%)(\mathrm{N}=116)$

\begin{tabular}{|l|c|c|}
\hline \multicolumn{2}{|c|}{ Girls (N=103) } & Boys (N=13) \\
\hline VERBAL sexual harassment & 44 & 62.5 \\
\hline Experienced once & 55 & 37.5 \\
\hline Experience often & 1 & - \\
\hline Continual experience & & \\
\hline Sexual GESTURES & 30 & 20 \\
\hline Experienced once & 66 & 80 \\
\hline Experience often & 4 & - \\
\hline Continual experience & & 100 \\
\hline PHYSICAL sexual harassment & 71 & - \\
\hline Experienced once & 28 & - \\
\hline Experience often & - & \\
\hline Continual experience & & \\
\hline
\end{tabular}

\section{A. VERBAL SEXUAL HARASSMENT}
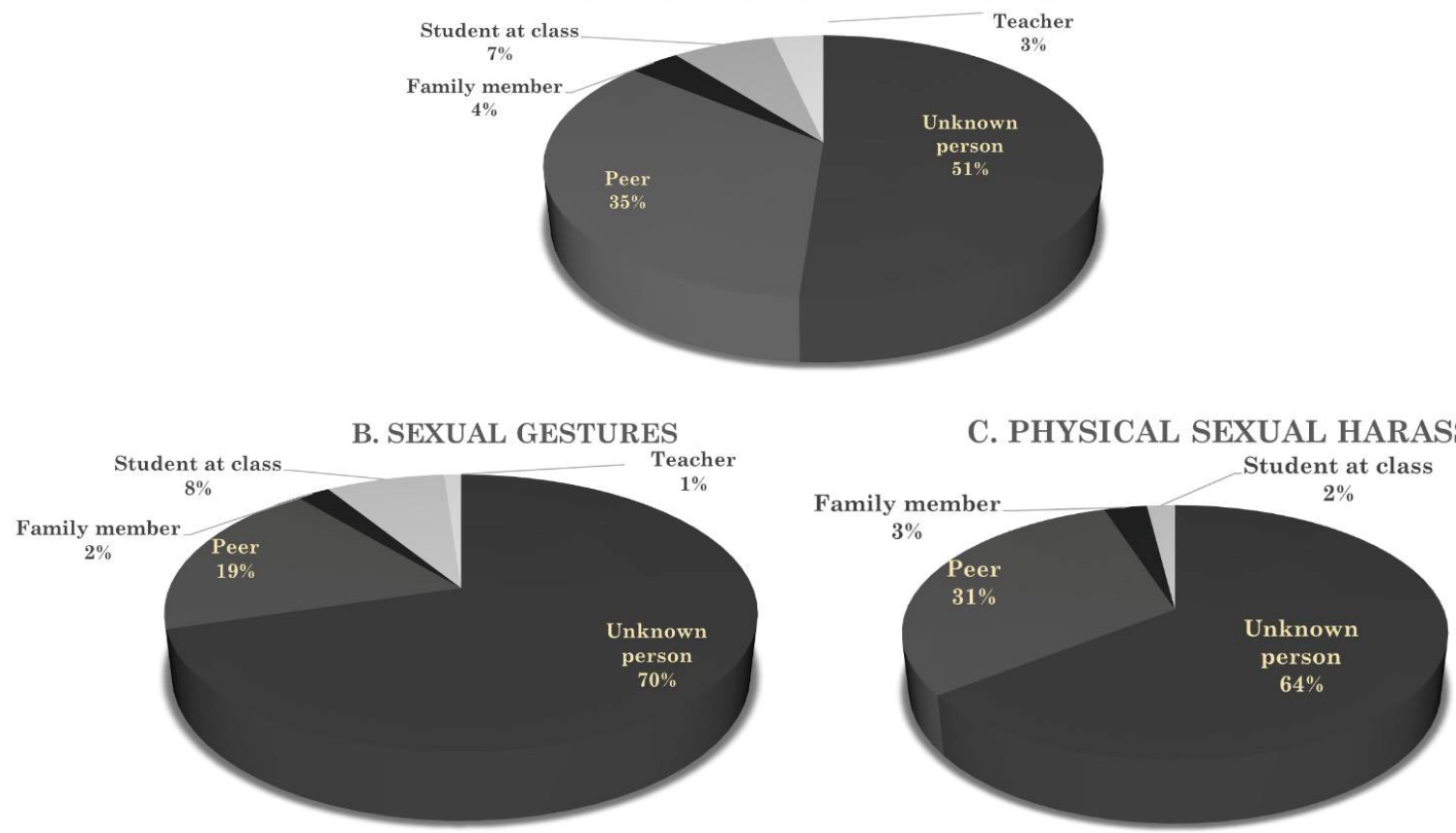

Figure 1. Sexual harassment by form and perpetrator, in percentage $(\mathrm{N}=116)$.

(A) Verbal sexual harassment by perpetrator, in percentage.

(B) Sexual gestures by perpetrator, in percentage.

(C) Physical sexual harassment by perpetrator, in percentage. 


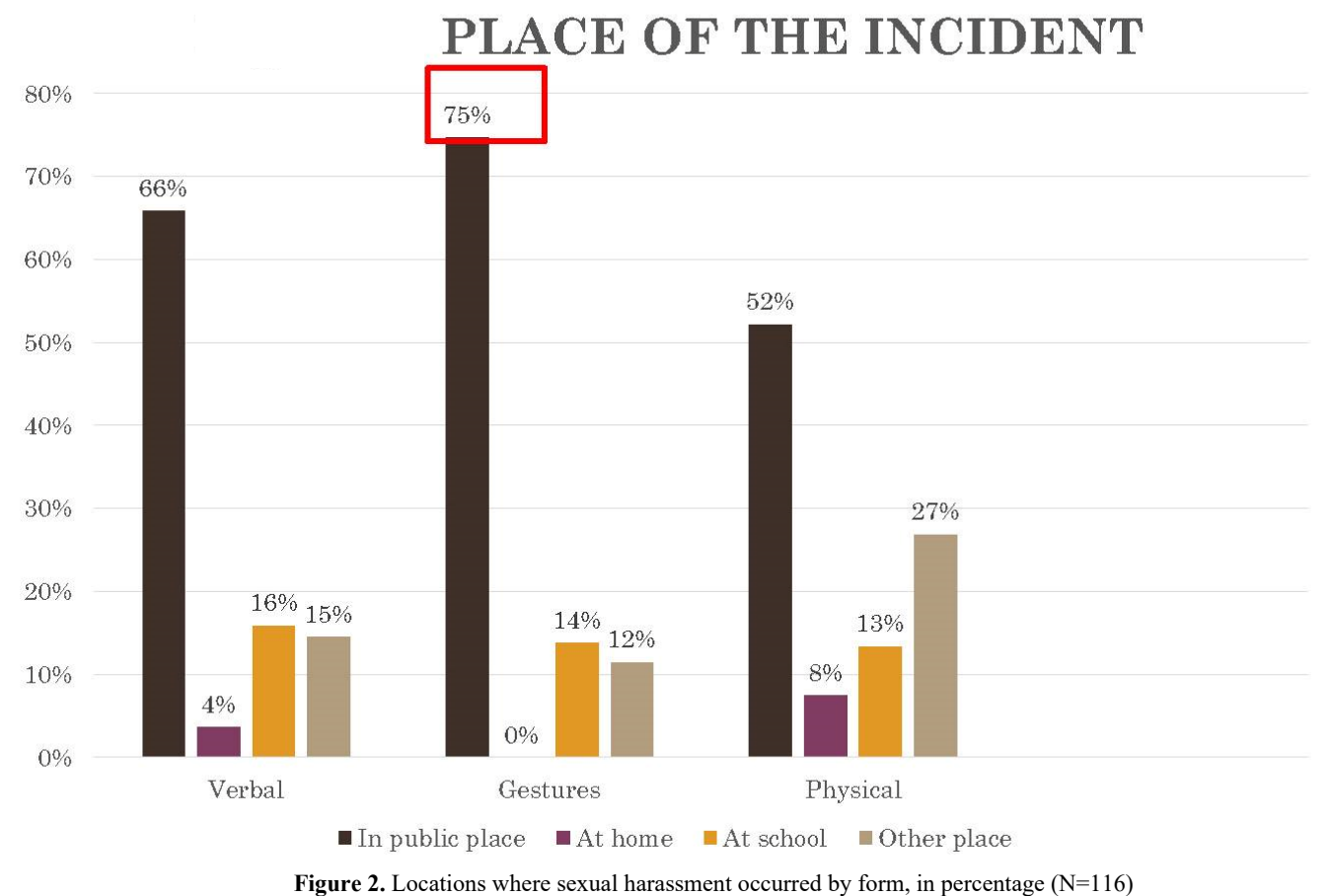

feelings evaluation median was 7,0 points (MIN-2; MAX-10). Evaluating subjective experience severity of sexual gestures, boys gave minimum 3 and maximum 5 points and a median was 4,0 , while girls evaluated from 1 to 10 , but a median was 7,0 . As could be predicted, the biggest point were given to physical unwelcome experience: boys reported their experienced physical sexual harassment incidents subjectively unpleasant from 3 to 10 point and a median was 6,5 , while girls - from 1 to 10 and the median was 9,0 out of ten!

In the last section of our questionnaire quantitative and qualitative data on personal evaluations of impact on three fields of physiological functioning was collected: on mood, sleeping and eating. For our great surprise, $50 \%$ of participants reported that experienced sexual harassment had some impact on their mood and emotions. This impact they evaluated in 7,0 median (MIN-1; MAX-10; SD-2,245). Here few descriptions of subjective experiences given by the participants are introduced:

I had permanent unpleasant thoughts about the incident, I used to rethink the incident and my reaction to what was happening and it used to make me sad.

I felt bad and unsafe. It was difficult to talk to older men for a while.

I feel bad while going on a bus, I try to observe atmosphere and all people on a bus and I am much more careful now, however, I still do not feel safe there.

I feel very bad about that accident. It is scary to walk alone at night in places where is dark.

Students also evaluated subjectively observed impact on their sleep. $11 \%$ indicated that they experience some kind of impact on their sleep, which was evaluated a 5,0 median (MIN-1; MAX-10; SD-2,148). Here are few examples of qualitative answers on observed impact on sleep:

I think I have lots of dreams, analogical to the incident.

Often I cannot fall asleep, because I think about what happened.
Often I cry in bed and cannot fall asleep.

$9 \%$ of participants reported that they observed some eating changes after the sexual harassment. Their experience severity median was 5,0 (MIN-2; MAX-8; SD-2,040). Here are few descriptions of the reported changes:

I started eating less from that incident, I was on a diet for a while.

I used to eat more because of stress and sadness.

\section{Discussion}

Sexual harassment is a common and widespread phenomenon. More than a half of adolescents aged 16-19 have reported unwelcome and unpleasant sexual comments, jokes, gestures or physical contact. Girls tend to be victims of this pattern significantly more often. More than three quarters of them reported experience of any form of sexual harassment. Though, $18 \%$ of boys indicated experiencing sexual harassment, according to other studies, this tend to grow with time [8].

Unlike previous researches, showing verbal sexual harassment being a major form of unwelcome sexual behavior [2,4,8], our research raised the hypothesis that the most common form of sexual harassment at the age of 16 to 19 is nonverbal communication or sexual gestures. However, this form is most common only when the victim is a girl, whereas boys tend to experience unpleasant verbal sexual behavior more often than sexual gestures.

Previous studies have highlighted the problem of peer sexual harassment and sexual harassment at schools $[1,2,4,10]$. Culture Model of sexual harassment at schools predicts that unwelcome sexual behavior by peers is commonplace, while sexual harassment by teachers is rare and restricted to isolated incidents $[4,8]$. The results of this pilot study shows that around one third of harassers are adolescents, often friends or students at school, whereas teachers are responsible only for 1-3\% of cases; furthermore, more than a half of unpleasant sexual behaviors are perpetrated by unknown people. 
It was known that girls are used to take sexual harassment more serious and might have more consequences on adaptation and health in the future $[4,9]$. Our data also revealed some differences between genders when evaluating subjective feelings about the sexual harassment: girls reported feeling more unpleasant about the incident than boys, and it did not depend on the form of sexual harassment. Not surprisingly, they felt the most uncomfortable about unwelcome physical sexual behavior.

Finally, we were surprised to find that sexual harassment, from a subjective view, affects over a half of victims' mood and emotions. Adolescents tend to rethink the incident and their own actions. They feel sad because of the incident and become apprehensive in suchlike situations. $11 \%$ of victims report having sleep disturbances and nightmares and $9 \%$ - the change of their eating habits (eating less, lower appetite, turning to diets, or overeating) and this needs to be explored more broadly.

\section{Implications}

Considering that the prevalence of sexual harassment has exceeded $50 \%$ among high school students, these kind of researches are highly important when planning future prospects of primary and secondary prevention programs and building secure and free of harassment environment for adolescents. Although sexual harassment is most common in public places when a perpetrator is an unknown person, it is alarmingly spread in important social environments, such as school and home. More than one tenth of reported cases took place at school, most often pupils were being harassed by another adolescent, and $4 \%$ of verbal and $8 \%$ of unpleasant physical sexual incidents has been reported to have happened at home. It reflects a Culture Model of sexual harassment which might be prevented by primary prevention and also social intervention programs.

Since $50 \%$ of respondents reported that they have experienced sexual harassment had some impact on their mood and emotions, and $11 \%$ of participants indicated that they observed some sleep disturbances associated to unwelcome and unpleasant sexual behavior in their past, these physiological disturbances could help to detect adolescents, who might need some professional help and specialized interventions to helping to cope with harassing experience and its burden.

\section{Acknowledgement}

To administration and all the teachers who kindly helped us to plan and carry out our research at school, and most importantly to the participants of the research for their time, honesty and patience.

\section{References}

1. American Association of University Women Educational Foundation (1993) Hostile hallways: The AAUW survey on sexual harassment in America's schools, Washington, DC.

2. American Association of University Women Educational Foundation (2001) Hostile hallways: Bullying, teasing and sexual harassment in school, Washington, DC.

3. Fineran S, Bennett L (1998) Teenage peer sexual harassment: implications for social work practice in education. Soc Work 43: 55-64. [Crossref]

4. Timmerman G (2003) Sexual Harassment of Adolescents Perpetrated by Teachers and by Peers: An Exploration of the Dynamics of Power, Culture, and Gender in Secondary Schools 48: 237-243.

5. Sarah K Murnen, Linda Smolak (2000) The Experience of Sexual Harassment Among Grade-School Students: Early Socialization of Female Subordination. Sex Roles 43: $1-17$.

6. Teresa Abada, Feng Hou, Bali Ram (2008) The effects of harassment and victimization on self-rated health and mental health among Canadian adolescents. Social Science \& Medicine 67: 561-564.

7. James E Gruber, Susan Fineran (2007) The Impact of Bullying and Sexual Harassment on Middle and High School Girls. Violence Against Women 13: 627-643.

8. American Association of University Women Educational Foundation (2011) Crossing the Line: Sexual Harassment at School," AAUW, Washington, DC.

9. Chesire DJ (2004) Test of an integrated model for high school sexual harassment (Doctoral dissertation).

10. Chiodo D, Wolfe DD, Crooks C, Hughes R, Jaffe P (2009) Impact of Sexual Harassment Victimization by Peers on Subsequent Adolescent Victimization and Adjustment: A Longitudinal Study. J Adolesc Health 45: 246-252.

Copyright: (C2018 Grigentyte G. This is an open-access article distributed under the terms of the Creative Commons Attribution License, which permits unrestricted use, distribution, and reproduction in any medium, provided the original author and source are credited. 